\title{
Motivation for Psychotherapy and Illness Beliefs in Turkish Immigrant Inpatients in Germany: Results of a Cultural Comparison Study
}

\author{
Hanna Reich • Luisa Bockel • Ricarda Mewes
}

Received: 13 May 2014 /Revised: 7 August 2014 / Accepted: 22 August 2014 / Published online: 1 October 2014

(C) W. Montague Cobb-NMA Health Institute 2014

\begin{abstract}
Objective Some immigrant populations, for instance, Turkish immigrants, suffer from worse mental health than the general population. Moreover, psychotherapeutic treatment does not work well in this group. This might be explained by lower motivation for psychotherapy and particular illness beliefs as important early predictors of treatment outcome. We investigate differences in these predictors between Turkish immigrant inpatients and inpatients without a migration background and evaluate whether particular illness beliefs have a negative impact on motivation for psychotherapy.

Method Turkish immigrant inpatients and inpatients without a migration background $(N=100)$, suffering from depressive disorder, somatoform disorder, and/or adjustment disorder, completed questionnaires assessing motivation for psychotherapy, depressive and somatic symptoms, illness perception, illness-related locus of control, and causal illness attributions. Results Despite a higher symptom burden, motivation for psychotherapy was lower in Turkish immigrant inpatients than in inpatients without a migration background $(d=0.54)$. This was fully explained by stronger beliefs in supernatural causes of illness and higher fatalistic-external illness-related locus of control in the Turkish immigrant sample (mediation analysis; $R^{2}=0.27$ ).

Conclusions Turkish immigrants believe in supernatural or fatalistic causes of illness and fatalistic-external locus of control to a greater extent than German inpatients without a migration background. These beliefs reduce motivation for psychotherapy and need to be addressed in psychotherapeutic treatment in order to secure positive treatment outcomes.
\end{abstract}

\section{H. Reich $(\bowtie) \cdot$ L. Bockel $\cdot$ R. Mewes}

Division of Clinical Psychology and Psychotherapy, Department of

Psychology, University of Marburg, Gutenbergstrasse 18,

35032 Marburg, Germany

e-mail: hanna.reich@staff.uni-marburg.de
Keywords Turkish immigrants $\cdot$ Motivation for psychotherapy · Illness beliefs · Affective disorders . Somatoform disorders

Migration is a highly defining life event which can lead to mental distress. It constitutes an overall risk factor for psychiatric disorders $[1,2]$. In the initial period following migration, a "healthy immigrant effect" can be observed, i.e., the prevalence of common mental health problems is initially lower than in the general population [3]. However, the health of immigrants tends to worsen over time to match that of the general population $[4,5]$. Moreover, some immigrant groups, e.g., Turkish immigrants, suffer from poor mental health, as demonstrated by elevated prevalence rates of common mental disorders like depression, anxiety disorders, and somatoform disorders [6-9]. Yet, psychotherapeutic treatment in immigrant patients is considered to be more complex, and the outcome appears to be less favorable than in patients without a migration background [10-12]. In order to improve the mental health treatment of immigrant patients, the reasons for this poor outcome need to be investigated. Specifically, knowledge about early predictors is necessary, which help to identify immigrant patients at risk for an unfavorable treatment outcome.

Our study focuses on Turkish immigrant inpatients and inpatients without a migration background in Germany. Turkish immigrants represent the largest minority group in Germany [13] and are among the largest three immigrant populations in other European countries such as The Netherlands, Denmark, and Austria [14]. It has been shown that Turkish immigrant inpatients in Germany achieve worse mental health treatment results than inpatients without a migration background $[15,16]$. Lower motivation for psychotherapy in Turkish immigrants might provide one explanation for this. 
Treatment motivation has been shown to be an important predictor of treatment success in clinical samples without a migration background [17-20]. Openness to psychotherapy pretreatment generally predicts more positive treatment outcomes [21]. Similarly, it was demonstrated that patients with depressive symptoms who were highly motivated for psychotherapeutic interventions from the outset benefited significantly more from inpatient treatment than those who were less motivated [22]. Motivation for psychotherapy has also been shown to predict dropout from inpatient treatment [23]. First studies investigating non-clinical immigrant samples showed that attitudes towards psychotherapy were more negative in second-generation Turkish immigrants than in Germans [24]. In sum, low motivation for psychotherapy is a risk factor for and early predictor of unfavorable treatment outcome. However, studies on motivation for psychotherapy in clinical samples with a migration background are lacking.

Illness beliefs are important determinants of treatment motivation $[25,26]$. Cultural norms and values influence specific attitudes and beliefs about illness [27] and offer explanatory models for understanding illness [28, 29]. For example, Turkish immigrants in Great Britain were found to be more convinced that health was outside their immediate control than native British people [30]. Likewise, Turkish-born female immigrants in Sweden mostly regarded their own capacity to influence recovery as low [31]. Two studies found stronger external causal illness attributions in Turkish immigrant inpatients than in German inpatients without a migration background $[32,33]$. Another study showed that types of attributions were related to hope for future well-being in Turkish inpatients: Attributing illness to the self-familial area of influence increased hope, whereas attributing illness to the uncontrollable area of influence decreased hope [34]. We assume that some illness beliefs that are prominent in Turkish immigrants, such as external locus of control and external illness attribution, reduce motivation for psychotherapy. To the best of our knowledge, no empirical study has tested this assumption to date.

In sum, treatment motivation is of crucial importance for treatment success. Motivation for psychotherapy in Turkish immigrants may be lower than in inpatients without a migration background. However, this effect has not yet been studied in a clinical sample. Furthermore, the reasons for the assumed lower motivation for psychotherapy are unclear. This is particularly important, as inferior outcomes of mental health interventions in immigrant populations can result in persistent individual suffering, as well as high societal costs. Understanding the reasons for reduced motivation for psychotherapy may help to identify individuals at risk for unfavorable outcomes and provide valuable information for the development of specific interventions to enhance treatment motivation in Turkish immigrant inpatients.
Notwithstanding the importance of motivation for psychotherapy, the concept of motivation itself is multifaceted and has been poorly defined in the literature $[25,35]$. Therefore, with the aim of avoiding ambiguities, we wish to make some specifications according to the model of psychotherapy motivation proposed by Drieschner and colleagues [25]: They differentiate between internal determinants of treatment motivation (e.g., problem recognition, level of suffering) and motivation to engage in treatment, which is specifically directed to a specific behavior, i.e., treatment engagement. The aspects of treatment motivation assessed in the present study are expectations of healing due to psychotherapeutic treatment, the type of role which the patient expects to play, attitudes towards psychotherapy, and previous experiences with psychotherapy. These concepts can be allocated to the internal determinants of treatment motivation in Drieschner's model of psychotherapy motivation.

In the present study, we examine the following hypotheses: (1) Turkish immigrant inpatients are less motivated for psychotherapy than German inpatients without a migration background. (2) Illness-related fatalistic-external locus of control, fatalistic causal illness attributions, and supernatural causal illness attributions are more pronounced among Turkish immigrant inpatients than in German inpatients without a migration background. (3) Lower motivation for psychotherapy in Turkish immigrant inpatients than in inpatients without a migration background (hypothesis 1) is mediated by illnessrelated fatalistic-external locus of control, fatalistic causal illness attributions, and supernatural causal illness attributions.

\section{Subjects and Methods}

\section{Sample}

A total of $n=50$ Turkish immigrant inpatients and $n=50$ German inpatients without a migration background participated in the study. All participants were inpatients in psychiatric treatment in Germany and had been diagnosed with depressive disorder, somatoform disorder, and/or adjustment disorder by experienced clinical staff. Demographic data of participants are presented in Table 1. In both subgroups, about twothirds of the participants were female, and the mean age was approximately 46 years. Turkish immigrant inpatients had received fewer years of education than inpatients without a migration background (7.42 vs. 11.06 years) and were less likely to be in employment ( $34 \%$ vs. $70 \%$ ). The difference in educational levels between Turkish immigrants in Germany and Germans without a migration background reflects actual differences at the population level [36]. According to the microcensus of the Federal Statistical Office and the Berlin Institute for Population and Development, Turkish 
Table 1 Study sample characteristics

\begin{tabular}{|c|c|c|c|c|c|c|}
\hline \multirow[b]{2}{*}{ Variable } & \multicolumn{2}{|c|}{ Turkish immigrant inpatients $^{\mathrm{a}}$} & \multicolumn{2}{|c|}{$\begin{array}{l}\text { Inpatients without a migration } \\
\text { background }^{\text {a }}\end{array}$} & \multirow[b]{2}{*}{$t$} & \multirow[b]{2}{*}{$p$} \\
\hline & $\mathrm{M}(\mathrm{SD})$ & Range & $\mathrm{M}(\mathrm{SD})$ & Range & & \\
\hline Age & $46.4(9.1)$ & $18-61$ & $45.9(8.9)$ & $24-65$ & -0.30 & 0.765 \\
\hline Education $^{\mathrm{b}}$ & $7.4(3.1)$ & $0-13$ & $11.1(1.7)$ & $9-15$ & 7.25 & $<0.001$ \\
\hline Severity of depressive symptoms (PHQ-9) & $18.0(5.4)$ & $5-27$ & $13.6(6.6)$ & $2-26$ & -3.63 & $<0.001$ \\
\hline \multirow[t]{2}{*}{ Severity of somatoform symptoms (PHQ-15) } & $14.0(4.8)$ & $4-24$ & $9.1(4.9)$ & $1-20$ & -5.09 & $<0.001$ \\
\hline & & & & & $\chi^{2}$ & $p$ \\
\hline Females & $66 \%$ & & & $62 \%$ & 0.17 & 0.677 \\
\hline Employment ${ }^{\mathrm{c}}$ & $34 \%$ & & & $70 \%$ & 12.98 & $<0.001$ \\
\hline
\end{tabular}

immigrants are the least integrated of all immigrant groups in Germany. This is apparent in high proportions of persons with no or only basic educational degrees, high rates of unemployed persons or housewives, and a great number of persons being dependent on social welfare $[37,38]$. Turkish immigrant inpatients in the present study reported a higher intensity of depressive and somatic symptoms than inpatients without a migration background, as indicated by higher scores on the PHQ-9 and PHQ-15 (see Table 1).

The majority of the Turkish immigrant inpatients were born in Turkey $(84 \%$ ) and had been living in Germany for about 31 years. Turkish immigrant inpatients born in Germany (16\%) were second-generation immigrants with both parents born in Turkey. Of the sample of Turkish immigrant inpatients, $6 \%$ had a temporary residence permit; $68 \%$ had a permanent residence permit, and $26 \%$ had a German passport. Their selfreported German language proficiency was as follows: $2 \%$ native speaker, $12 \%$ very good, $26 \%$ good, $20 \%$ intermediate, and $40 \%$ poor. $82 \%$ of the Turkish immigrant inpatients chose to respond to the Turkish versions of the questionnaires.

\section{Procedure}

In this cross-sectional study, data were collected between September 2011 and January 2012 in five psychiatric hospitals in the Federal State of Hessen, Germany. In Germany, all patients are referred to inpatient treatment by their family doctor and/or a psychiatrist. Inclusion criteria for study participation were: age 18-65 years, either Turkish migration background or no migration background at all, and a clinical diagnosis of depressive disorder, somatoform disorder, or adjustment disorder. These mental illnesses were selected because they are the most widely observed in Turkish immigrant inpatients in Germany [39]. Comorbidities were allowed. Exclusion criteria were: psychotic or manic symptoms, substance abuse, and neurodegenerative diseases. Inpatients who met the study criteria were asked if they would be willing to participate voluntarily in the study. All participating patients signed informed consent forms. They provided demographic data and completed several self-rating questionnaires as described below. At the time of data collection, $27 \%$ of the participants had been in treatment for less than a week, $54 \%$ for 1 to 4 weeks, and $19 \%$ for more than 4 weeks. Questionnaires were administered individually. All instruments were provided in German and Turkish versions. A bilingual research assistant was present to help participants at all times and also read out questions to the participants if they were illiterate (this applied to some Turkish women). Samples were matched according to age, sex, and clinic site. No data regarding non-participation were obtained in this study. The study was approved by the local Ethics Committee at the Institute of Psychology at Philipps-University, Marburg.

\section{Measures}

The instruments used in the present study are outlined below. The instruments FMP, PHQ-15, IPQ-R Causal Illness Attributions, and KKG were not available in Turkish. Therefore, they were translated into the Turkish language following the forward- and backward-translation method [40].

Psychotherapeutic Treatment Expectations and Openness to Psychotherapy Scales (FMP) This German-language questionnaire [41] aims to assess patients' motivation for psychotherapy. In the present study, we operationalize motivation for psychotherapy according to four different aspects: expectations of healing due to psychotherapeutic treatment, the type of role the patient expects to play, attitudes towards psychotherapy, and previous experiences with psychotherapy. Consequently, we used the first two scales of the FMP (28 items), i.e., Psychotherapeutic Treatment Expectations and Openness to Psychotherapy. 
Response categories range from 1 ("not at all") to 5 ("fully agree"). The sum score of all 28 items ranged from 28 to 140 and was used as an indicator of the motivation for psychotherapy. Symptom-related suffering (scale 3 of the FMP) and causal illness attributions (scale 4) were assessed by other instruments (see below). The Turkish version was translated as described above.

\section{The Patient Health Questionnaire Depressive and Somatic} Symptom Scales (PHQ-9, PHQ-15) The severity of depressive and somatic symptoms was measured with the PHQ9 and PHQ-15, respectively [42-44]. Nine items of the PHQ assess depressive symptoms (PHQ-9), and the scale has shown high sensitivity and specificity for the identification of depressive disorders [45, 46]. Response categories for these items range from 0 ("not at all") to 3 ("nearly every day"). For the present study, a continuous depression score was calculated, which consisted of the sum of the answers to the nine items, ranging from 0 to 27. The German and the Turkish versions of the PHQ-9 $[47,48]$ are available from the Pfizer PHQ-screener website [49]. A recent study showed acceptable reliability and validity for the Patient Health Questionnaire in the Turkish population [50].

Somatic complaints were screened with the PHQ-15 subscale, a checklist of 15 somatic symptoms typical for primary care patients, which are often medically unexplained [43, 44, 51]. The PHQ-15 assesses a broad range of pain symptoms, especially pain in the stomach, back pain, headache, chest pain, pain in extremities or in joints, pain associated with menstruation, and pain during sexual intercourse. The latter two items were not used in the present study, since they were considered as culturally inappropriate for Turkish immigrants. For the last 4 weeks, the severity of each symptom can be rated from 0 ("not bothered at all") to 2 ("bothered a lot"); the total score ranges from 0 to 30 . The German version is available online [49]; the Turkish version was translated as described above.

The Brief Illness Perception Questionnaire (Brief IPQ) The Brief IPQ [52] assesses the subjective illness concept and the cognitive representations about illness using one item for each scale of the more detailed IPQ-R [53], namely consequences, timeline, personal control, treatment control, identity, concern, understanding, and emotional response. Response categories for these items ranged from 0 to 10 , with endpoints being labeled according to the questions (e.g., for the question "How much does your illness affect your life?", the labeled endpoints are 0 "no affect at all" to 10 "severely affects my life"); the sum score for the Brief IPQ ranges from 0 to 80 , with higher scores indicating a more pessimistic and threatening illness perception. Turkish and German versions of the Brief IPQ were available $[54,55]$.
The Revised Illness Perception Questionnaire (IPQ-R) Causal Illness Attributions Scale For the assessment of causal illness attributions, we chose not to use the open response format provided by the Brief IPQ, but to administer closed-format items. For this purpose, the scale 'causal illness attributions' of the IPQ-R [53] plus three culture-specific items derived from the work of Kizilhan [33] were used. These describe spiritual and supernatural illness attributions, namely "God's will", "curse/ enchantment", and "evil spirits". Patients were asked to rate how strongly they believe in a certain cause of illness, with response categories ranging from 1 ("not at all") to 5 ("fully agree"). A German version was available [56]; the Turkish version was translated as described above. Exploratory factor analysis revealed five factors of illness attributions that were used for further analysis: psychological cause ("worry", "emotional state", "family problems", "negative mental attitude"), stress-related cause ("stress", "overwork"), biological cause ("somatic/biomedical", "heredity", "pollution or other external influences"), fatalistic cause ("bad luck", "destiny"), and supernatural cause ("God's will", "curse/ enchantment", "evil spirits").

Locus of Control Inventory for Illness and Health $(K K G)$ While the Brief IPQ provides only two items asking about personal and treatment control, the German-language questionnaire KKG [57] extends the assessment and measures three dimensions of illness-related locus of control (LOC), namely beliefs in internal, social-external, and fatalistic-external illness-related LOC. Each dimension is assessed by seven items, with response options ranging from 1 ("not at all") to 6 ("fully agree"); dimensional scores range from 7 to 42. Higher scores indicate greater conviction regarding a particular LOC dimension. The Turkish version was translated as described above.

\section{Statistics}

Statistical analyses were carried out using SPSS 19 for Windows. Prerequisites for data analyses were checked, and data were normally distributed. Less than $5 \%$ of values were missing completely at random (Little's MCAR test: n.s.), and missing values were imputed using the expectation maximum-method employing the SPSS missing value tool.

For sample comparisons of key dependent variables, data were analyzed for each questionnaire using univariate or multivariate analysis of variance as appropriate. Due to the fact that sample differences in education and employment reflect natural characteristics of differences between these groups in the German population, no covariates were included in this step of analysis to avoid over-adjustment. Standardized effect sizes are given as Cohen's $d$ [58]. The $95 \%$ confidence 
intervals for Cohen's $d$ were calculated following the recommendations of Grissom and Kim [59]. Confidence intervals that do not contain 0 provide evidence, at the two-tailed 0.05 level of statistical significance, that the effect in the population does not equal 0 [59], i.e., that there actually is a difference between Turkish immigrant inpatients and German inpatients without a migration background in the construct in question (e.g., motivation for psychotherapy).

Next, a hierarchical multiple regression analysis was conducted to examine which demographic, symptom severity, or illness beliefs variables predicted motivation for psychotherapy in the examined group as a whole (Turkish immigrant inpatients and inpatients without a migration background as one group). To control for the influence of migration background, this variable was entered in step 1, followed by further demographic variables in step 2, depressive and somatic symptom intensity in step 3, and illness beliefs in step 4 . The $\beta$-coefficients given in the results section are partial regression coefficients. As an estimator of effect size, each partial regression coefficient estimates the change in the dependent variable for each unit increase in the corresponding independent variable when all other dependent variables are statistically held constant, i.e., estimating the "pure" contribution [59]. Means of $\beta$-coefficients $=0$, and standard deviations $=1$, as in $z$-scores.

Finally, we ran a multiple-mediation model to test the hypothesis that lower motivation for psychotherapy in Turkish immigrant inpatients is mediated by illness-related fatalisticexternal locus of control, fatalistic causal illness attributions, and supernatural causal illness attributions. Mediation exists when a predictor ( $X$ : Turkish migration background vs. no migration background) affects a dependent variable ( $Y$ : motivation for psychotherapy) indirectly through at least one intervening variable or mediator ( $M$ : illness beliefs, see Fig. 1). Mediation analysis helps to establish causal relationships between variables and explain how or by what means a causal effect occurs [60].

According to Preacher and Hayes [60], special qualities of multiple mediation (instead of various univariate mediation tests) include (a) the analysis of the total indirect effect, i.e., an overall mediation effect of all the mediators under investigation and (b) the ability to determine the unique mediating effect of a specific variable within a single model, controlling for the presence of other mediators. Bootstrap analysis, a nonparametric sampling procedure, was used to test the significance of the indirect effects. Bootstrap analysis has the advantage of greater statistical power without assuming multivariate normality in the sampling distribution, allowing a parsimonious and precise analysis of multiple mediators [60, 61]. For our analysis, we used the SPSS macro designed for multiple mediation analysis by Preacher and Hayes [60]. Five thousand bootstrap samples were obtained; confidence intervals were bias-corrected and accelerated.

\section{Results}

Intercorrelations Among Study Variables

A correlation matrix was generated to examine the bivariate relationship between each psychosocial and demographic variable in the study and to check for multicollinearity. Various study variables showed correlations in the expected direction (see Table 2). However, the pattern of relationships of these variables was mainly modest in size, i.e., multicollinearity was not found.

Sample Differences in Motivation for Psychotherapy, Illness-Related Locus of Control, and Causal Illness Attributions

Turkish immigrant inpatients were less motivated for psychotherapy than inpatients without a migration background $(p=$ 0.008 ), with the difference equating to a medium effect size ( $d$ $[\mathrm{CI}]=0.54[0.14 ; 0.94]$, Table 3). Moreover, Turkish immigrant inpatients perceived their illness as more impairing and less controllable than did inpatients without a migration background ( $p=0.001$ ), with the difference equating to a medium to large effect size $(d[\mathrm{CI}]=0.7[0.3 ; 1.1])$. Regarding illnessrelated LOC, Turkish immigrant inpatients had lower internal control beliefs $(p<0.001)$ and higher fatalistic-external control beliefs $(p=0.001)$ than inpatients without a migration background, with both differences equating to medium to large effect sizes $(d[\mathrm{CI}]=0.81[0.4 ; 1.22]$, and $d[\mathrm{CI}]=0.7$ $[0.3 ; 1.1]$, resp.). There was no difference in social-external LOC. Turkish immigrant inpatients were more convinced of a fatalistic $(p=0.002, d[\mathrm{CI}]=0.66[0.26 ; 1.06]$, i.e., medium effect size) or supernatural cause of their illness $(p<0.001, d$ $[\mathrm{CI}]=1.03[0.61 ; 1.45]$, i.e., large effect size) than inpatients without a migration background, while there were no differences between samples regarding beliefs in psychological, stress-related, or biological causes of illness.

\section{Predictors of Motivation for Psychotherapy}

Through the application of a hierarchical multiple regression analysis, it is possible to test the hypothesis that illness beliefs have an additional and unique explanatory power regarding motivation for psychotherapy going beyond demographic variables and symptom severity. The results showed that only migration background and illness beliefs were significant predictors of motivation for psychotherapy ( $p \leq 0.01$, and $p \leq$ 0.05 , respectively, see Table 4).

The migration background accounted for $7 \%$ of the variance in motivation for psychotherapy, and illness beliefs explained a further $20 \%$. In particular, fatalistic-external LOC $(\beta=-0.26, p=0.042)$ and supernatural causal illness attribution $(\beta=-0.33, p=0.007)$ showed a significant negative 
Fig. 1 Multiple-mediation model for the influence of migration background on motivation for psychotherapy mediated by illness beliefs, tested on the basis of Preacher and Hayes [60]. $X$ independent variable, migration background; $M_{l}-M_{9}$ proposed mediators; $M_{1}$ illness perception; $M_{2}$ internal locus of control; $M_{3}$ social-external locus of control; $M_{4}$ fatalistic-external locus of control; $M_{5}$ psychological illness attribution; $M_{6}$ stress-related illness attribution; $M_{7}$ biological illness attribution; $M_{8}$ fatalistic illness attribution; $M_{9}$ supernatural illness attribution; $Y$ dependent variable, motivation for psychotherapy

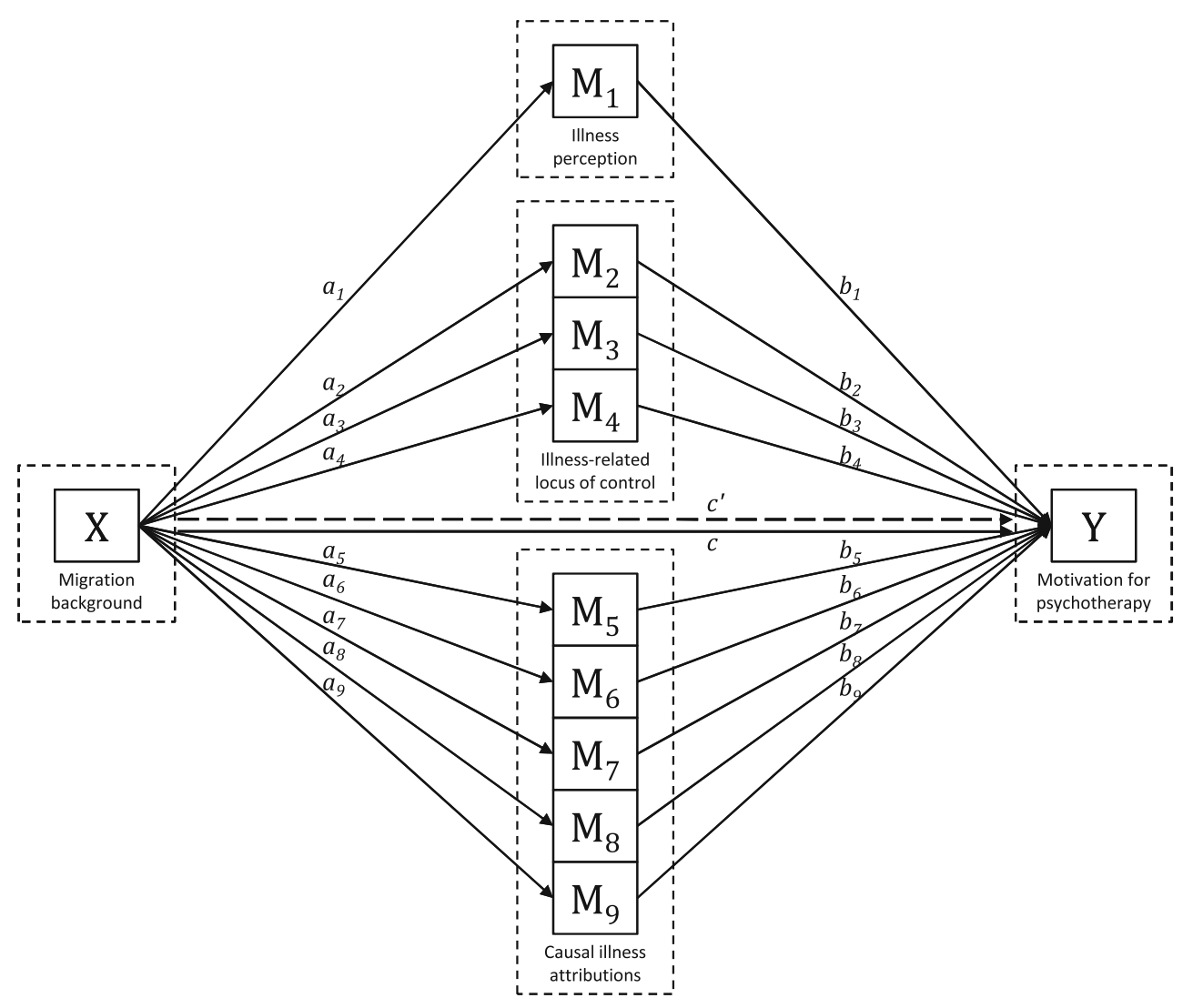

impact on motivation for psychotherapy, i.e., the stronger the fatalistic-external LOC and the supernatural causal illness attribution, the lower the motivation for psychotherapy.

Mediating Effects of Illness Beliefs on Motivation for Psychotherapy

The hierarchical multiple regression analysis demonstrated the major influence of illness beliefs on motivation for psychotherapy. Based on this, a multiple mediation model was tested in order to examine the hypothesis that lower motivation for psychotherapy in Turkish immigrant inpatients is mediated by illness-related fatalistic-external locus of control, fatalistic causal illness attributions, and supernatural causal illness attributions. The multiple mediation model (see Fig. 1 and Table 5) showed a significant fit, and the proportion of explained variance was medium to high in line with Cohen [58] $\left(R^{2}=0.27\right.$, Adj. $\left.R^{2}=0.19, F(10,89)=3.28, p=0.001\right)$. The total indirect effect of illness beliefs was significant, as indicated by the $95 \%$ bias-corrected and accelerated bootstrapping confidence interval that did not contain zero (95\% BCA CI $=[-12.96 ;-2.67])$. The direct effect of a migration background on psychotherapy motivation (c' path) was no longer significant when illness beliefs were included in the model $(p>0.05)$. Consequently, the association between migration background and psychotherapy motivation was fully mediated by illness beliefs.

Examining the specific indirect effects of each mediator on the relationship between migration background and motivation for psychotherapy, fatalistic-external locus of control, and supernatural illness attribution were significant mediators $(95 \%$ BCA CI for $a b-$ path $=[-5.15 ;-0.57]$, and $=[-8.30$; $-0.49]$, resp.). Turkish migration background was positively related to fatalistic-external locus of control $(B=4.56, p \leq$ $0.001)$ and to supernatural illness attributions $(B=0.99, p \leq$ $0.001)$, which in turn were negatively related to motivation for psychotherapy $(B=-0.50, p \leq 0.05$, and $B=-3.73, p \leq 0.01$, resp.).

\section{Discussion}

The current study focused on the influence of migration background on early predictors of treatment outcome, namely motivation for psychotherapy and illness beliefs. Motivation for psychotherapy was lower in Turkish immigrant inpatients than in German inpatients without a migration background. Turkish immigrant inpatients perceived their illness as less controllable by themselves and more controlled by fatalistic influences than did inpatients without a migration 


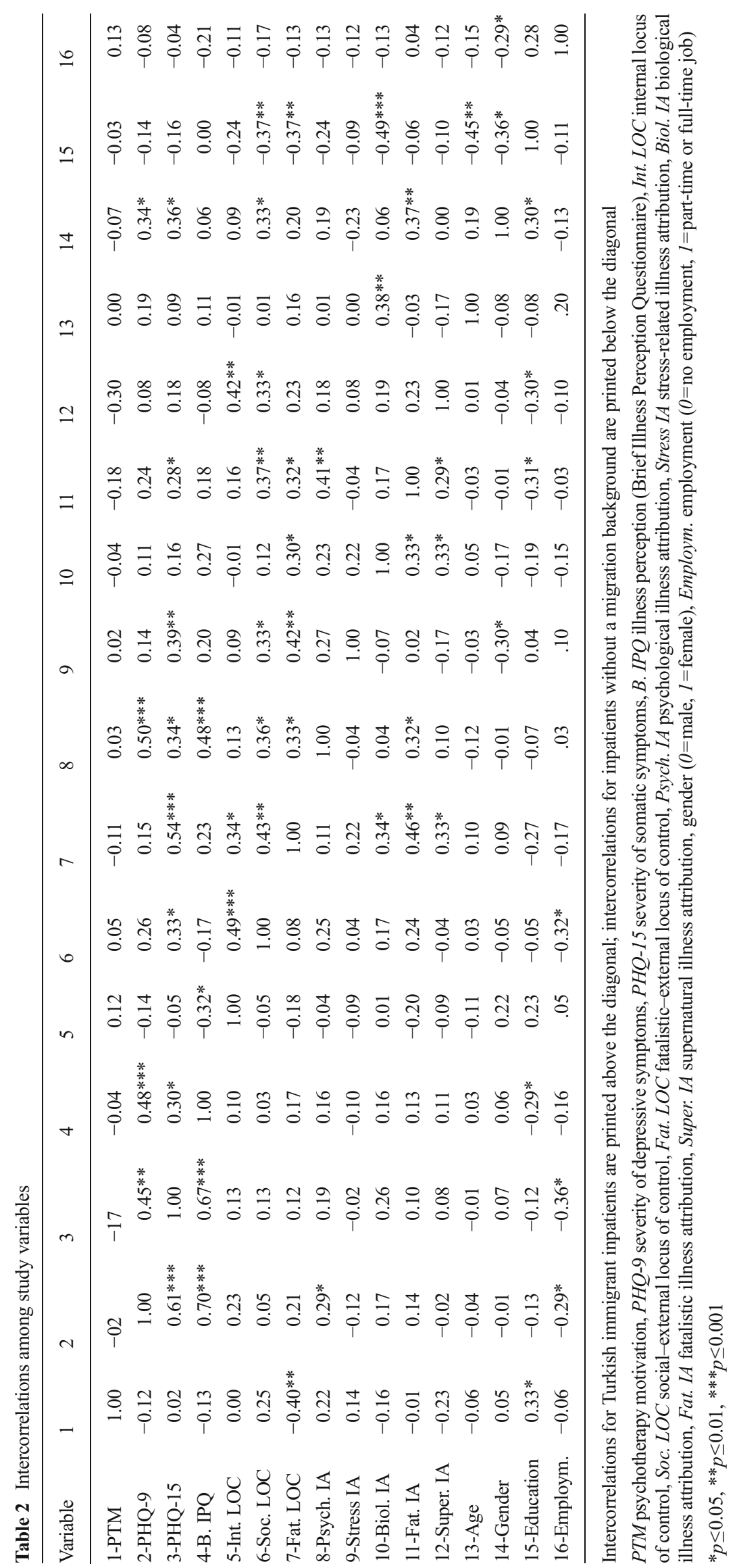


Table 3 Sample differences in motivation for psychotherapy, illness perception, locus of control, and causal illness attributions

\begin{tabular}{|c|c|c|c|c|c|c|c|}
\hline \multirow[b]{2}{*}{ Variable } & \multicolumn{2}{|c|}{ Turkish immigrant inpatients ${ }^{\mathrm{a}}$} & \multicolumn{2}{|c|}{ Inpatients without a migration background ${ }^{\mathrm{a}}$} & \multirow[b]{2}{*}{$F$} & \multirow[b]{2}{*}{$p$} & \multirow[b]{2}{*}{$d[C I]^{\mathrm{b}}$} \\
\hline & M & SD & M & SD & & & \\
\hline Motivation for psychotherapy & 101.4 & 13.2 & 107.8 & 10.7 & 7.24 & 0.008 & $0.54[0.14 ; 0.94]$ \\
\hline Illness perception & 55.4 & 11.6 & 47.2 & 12.2 & 11.90 & 0.001 & $0.7[0.3 ; 1.1]$ \\
\hline \multicolumn{8}{|l|}{ Locus of control } \\
\hline Internal & 23.2 & 8.3 & 28.6 & 4.6 & 16.32 & $<0.001$ & $0.81[0.4 ; 1.22]$ \\
\hline Social-external & 26.4 & 7.2 & 24.2 & 5.4 & 2.91 & 0.091 & $0.35[-0.04 ; 0.74]$ \\
\hline Fatalistic-external & 22.2 & 7.1 & 17.6 & 6.1 & 11.92 & 0.001 & $0.7[0.3 ; 1.1]$ \\
\hline \multicolumn{8}{|l|}{ Causal illness attributions } \\
\hline Psychological cause & 4.3 & 0.9 & 4.2 & 0.7 & 0.53 & 0.467 & $0.13[-0.26 ; 0.52]$ \\
\hline Stress-related cause & 4.2 & 1.0 & 4.4 & 0.8 & 1.19 & 0.279 & $0.22[-0.17 ; 0.61]$ \\
\hline Biological cause & 2.9 & 1.1 & 2.6 & 0.9 & 2.39 & 0.125 & $0.3[-0.09 ; 0.69]$ \\
\hline Fatalistic cause & 2.9 & 1.4 & 2.1 & 1.0 & 10.04 & 0.002 & $0.66[0.26 ; 1.06]$ \\
\hline Supernatural cause & 2.4 & 1.2 & 1.4 & 0.7 & 25.10 & $<0.001$ & $1.03[0.61 ; 1.45]$ \\
\hline
\end{tabular}

All means and standard deviations represent raw, nonstandardized scores

${ }^{\mathrm{a}} n=50$ each

${ }^{\mathrm{b}}$ Standardized effect size Cohen's $d$ [95\% confidence interval of Cohen's $d$ ]. According to Cohen (1988), $d=0.2$ can be interpreted as a small effect, $d=$ 0.5 as a medium effect, and $d=0.8$ as a large effect. Confidence intervals of Cohen's $d$ that do not include 0 provide evidence that there is a difference between the populations of Turkish immigrants and Germans without a migration background in the corresponding variable

Table 4 Hierarchical linear regression analysis for motivation for psychotherapy as dependent variable $(n=100)$

\begin{tabular}{|c|c|c|c|c|c|c|}
\hline \multirow[b]{2}{*}{ Predictor variables } & \multicolumn{6}{|c|}{ Dependent variable: motivation for psychotherapy } \\
\hline & $B$ & $\mathrm{SE} B$ & $\beta$ & Adj. $R^{2}$ & $\Delta R^{2}$ & $F$ \\
\hline Step 1: migration background ${ }^{\mathrm{a}}$ & -6.45 & 2.40 & $-0.26^{* *}$ & 0.059 & 0.069 & $7.24 * *$ \\
\hline Step 2: demographic characteristics & & & & 0.027 & 0.007 & 1.54 \\
\hline Age & -0.01 & 0.14 & -0.01 & & & \\
\hline Gender & -0.03 & 2.63 & 0.00 & & & \\
\hline Education & 0.34 & 0.53 & 0.08 & & & \\
\hline Employment & 0.96 & 2.70 & 0.04 & & & \\
\hline Step 3: symptom severity & & & & 0.010 & 0.004 & 1.15 \\
\hline Depressive symptoms & -0.04 & 0.22 & -0.02 & & & \\
\hline Somatic symptoms & -0.14 & 0.31 & -0.06 & & & \\
\hline Step 4: illness beliefs & & & & 0.140 & 0.199 & $2.01 *$ \\
\hline Illness perception & 0.01 & 0.14 & 0.01 & & & \\
\hline Internal locus of control & 0.26 & 0.20 & 0.15 & & & \\
\hline Social-external locus of control & 0.38 & 0.24 & 0.20 & & & \\
\hline Fatalistic-external locus of control & -0.47 & 0.23 & $-0.26^{*}$ & & & \\
\hline Psychological illness attribution & 3.07 & 1.84 & 0.19 & & & \\
\hline Stress-related illness attribution & 1.07 & 1.57 & 0.08 & & & \\
\hline Biological illness attribution & 0.66 & 1.35 & 0.05 & & & \\
\hline Fatalistic illness attribution & -0.85 & 1.17 & -0.09 & & & \\
\hline Supernatural illness attribution & -3.73 & 1.35 & $-0.33 * *$ & & & \\
\hline
\end{tabular}

$B$ unstandardized coefficients, SE $B$ standard error of unstandardized coefficients, $\beta$ standardized coefficients

${ }^{\mathrm{a}} 0$ Inpatients without a migration background, 1 Turkish immigrant inpatients

${ }^{*} p \leq 0.05,{ }^{*} p \leq 0.01$ 
Table 5 Multiple-mediation model for the influence of a migration background mediated by illness beliefs (proposed mediators, $\mathrm{M}_{1}-\mathrm{M}_{9}$ ) on motivation for psychotherapy (outcome)

\begin{tabular}{|c|c|c|c|c|c|c|c|c|}
\hline \multirow[t]{3}{*}{ Mediators } & \multicolumn{2}{|c|}{$\begin{array}{l}\text { Migration background to mediator } \\
\text { (a-path) }\end{array}$} & \multicolumn{2}{|c|}{$\begin{array}{l}\text { Mediator to outcome } \\
\text { (a-path) }\end{array}$} & \multicolumn{4}{|c|}{ Migration background to outcome ( $a b$-path) } \\
\hline & \multirow[t]{2}{*}{$P E$} & \multirow[t]{2}{*}{$S E$} & \multirow[t]{2}{*}{$P E$} & \multirow[t]{2}{*}{$S E$} & \multirow[t]{2}{*}{$P E$} & \multirow[t]{2}{*}{$S E$} & \multicolumn{2}{|c|}{$95 \%$ BCA CI } \\
\hline & & & & & & & Lower & Upper \\
\hline $\mathrm{M}_{1}$-illness perception & $8.19 * * *$ & 2.37 & -0.06 & 0.11 & -0.47 & 0.79 & -2.13 & 1.16 \\
\hline $\mathrm{M}_{2}$-internal locus of control & $-5.40 * * *$ & 1.34 & 0.26 & 0.19 & 1.39 & 1.10 & -3.79 & 0.55 \\
\hline $\mathrm{M}_{3}$-social-external locus of control & 2.17 & 1.27 & 0.31 & 0.21 & 0.67 & 0.65 & -0.13 & 2.86 \\
\hline $\mathrm{M}_{4}$-fatalistic-external locus of control & $4.56^{* * *}$ & 1.32 & $-0.50^{*}$ & 0.21 & $-2.27^{\text {sign. }}$ & 1.12 & -5.15 & -0.57 \\
\hline $\mathrm{M}_{5}$-psychological illness attribution & 0.11 & 0.15 & 2.76 & 1.73 & 0.31 & 0.58 & -0.51 & 1.89 \\
\hline $\mathrm{M}_{6}$-stress-related illness attribution & -0.19 & 0.18 & 1.25 & 1.40 & -0.24 & 0.40 & -1.67 & 0.23 \\
\hline $\mathrm{M}_{7}$-biological illness attribution & 0.31 & 0.20 & 0.43 & 1.21 & 0.13 & 0.51 & -0.60 & 1.69 \\
\hline $\mathrm{M}_{8}$-fatalistic illness attribution & $0.78^{* *}$ & 0.25 & -0.69 & 1.10 & -0.54 & 1.05 & -2.81 & 1.45 \\
\hline $\mathrm{M}_{9}$-supernatural illness attribution & $0.99 * * *$ & 0.20 & $-3.73 * *$ & 1.27 & $-3.68^{\text {sign. }}$ & 1.92 & -8.30 & -0.49 \\
\hline Total indirect effect & & & & & -7.48 sign. & 2.59 & -12.96 & -2.67 \\
\hline Direct effect $\left(c^{\prime}\right)$ & & & & & 1.02 & 3.04 & & \\
\hline Total effect $(c)$ & & & & & $-6.45^{* *}$ & 2.40 & & \\
\hline
\end{tabular}

Model summary for DV model: $R^{2}=0.27$, Adj. $R^{2}=0.19, F(10,89)=3.28, p=0.001 ; P E$ parameter estimate, $S E$ standard error of parameter estimate, $95 \%$ BCA CI $95 \%$ bias-corrected and accelerated bootstrapping confidence intervals for 5,000 bootstrap samples, ${ }^{\text {sign }}$ confidence interval does not contain zero, the indirect effect can be interpreted as significant; confidence intervals containing zero are interpreted as not significant

${ }^{*} p \leq 0.05,{ }^{* *} p \leq 0.01, * * * p \leq 0.001$

background. Furthermore, they were more convinced of fatalistic or supernatural causes of illness than inpatients without a migration background. In this respect, our initial hypotheses were supported. Differences between the samples in terms of illness beliefs explained differences in motivation for psychotherapy. Confidence intervals of the effect sizes for significant sample differences did not include 0, i.e., we can infer from the present data that there are actual differences in motivation for psychotherapy, illness perception, illness-related locus of control, and belief in supernatural or fatalistic causes of illness at the population level.

Since the concept of motivation for psychotherapy is multifaceted, making some specifications regarding our results will be helpful in terms of achieving a sophisticated understanding of the aspects according to which Turkish immigrant inpatients differ from the majority of German inpatients without a migration background. Our results indicate that Turkish immigrant inpatients have higher expectations of healing from medical treatment than from psychotherapeutic interventions. This is in line with a previous study [62] stating that Turkish immigrant outpatients with psychosomatic complaints are commonly treated by a general practitioner or with pharmacological treatment instead of a psychotherapist. Moreover, they request drugs more intensively and are more convinced of the effectiveness of medicines. Our results also indicate that Turkish immigrant inpatients are likely to expect to assume a passive role as a patient in psychotherapy. This is in line with findings that Turkish people hold cultural values that promote respect towards authority figures and do not question or challenge authorities [63]. Furthermore, our results indicate that previous experiences with psychotherapy are rare in Turkish immigrant inpatients, which is confirmed by studies about health care utilization [64-66].

Our study samples differed in their belief in supernatural and fatalistic causes of illness. In the German majority society, such beliefs are quite uncommon. Moreover, rural populations in Turkey were found to favor psychological, social, and medical explanations as causes of mental illness [67], and a community sample of Turkish immigrants in Australia believed more frequently in natural rather than supernatural causes of illness [68]. Nonetheless, beliefs regarding causes of mental illness range from traditional, supernatural attributions to biological attributions in the Turkish culture [69]. Integrating these results, we can assume that Turkish immigrants hold a broader range of causal illness attributions than Germans without a migration background.

Characteristics of the illness-related locus of control also varied considerably between the investigated samples. The emphasis on fatalistic-external locus of control in Turkish immigrant inpatients is in line with the literature [30, 63, 70]; less educated persons, in particular, believe that external factors control their lives [71].

Besides illness beliefs, the level of suffering is another possible predictor of motivation for psychotherapy [21, 72-74]. While our finding of higher symptom levels among Turkish immigrant inpatients is in line with other studies [39, 
75], it is noteworthy that this did not influence the motivation for psychotherapy. We can assume that the other factors (e.g., illness beliefs) had a stronger effect on decreasing the motivation for psychotherapy than suffering did on increasing it. On the other hand, the higher symptom scores in the Turkish immigrant inpatients might be explained by a culture-specific aspect of illness communication. Expressing distress, especially about somatic symptoms, and blatant suffering has been observed in immigrant populations before $[33,76,77]$. However, the equivalence of measures has not yet been established for the German and Turkish versions of the PHQ-9 and PHQ15. Therefore, caution is warranted when comparing mean scores of these instruments.

Sample differences in socio-demographic variables such as education and employment did not explain any differences in treatment motivation. However, lower education coincided with a stronger conviction regarding fatalistic locus of control in the Turkish immigrant sample, and with a lower motivation for psychotherapy, and fatalistic and supernatural illness attributions in the sample without a migration background. This demonstrates an unfavorable influence of low education in general, i.e., low education affected motivation for psychotherapy and illness beliefs negatively in both samples.

Although our samples provided a unique opportunity to compare motivation for psychotherapy and illness beliefs depending on migration background, there may be some limitations regarding the generalizability of our results. Specifically, our samples were rather small and solely included inpatients in psychotherapeutic treatment. No analysis was conducted on non-participants. In the immigrant sample, first-generation immigrants were overrepresented. Consequently, our results may not generalize to all Turkish immigrants (e.g., younger and often more highly educated "secondgeneration" immigrants; persons without mental disorder or persons who are not attending treatment). Additional studies comparing other important subgroups would help to gain a diversified understanding of the influences of a migration background, culture of origin, and culture of the host country on mental health beliefs. At this point, the application of qualitative methods, e.g., deep interviews, could also be very helpful to get more detailed information on motivation for psychotherapy and illness beliefs in culturally diverse inpatient populations. Analyses of demographic characteristics indicated that the samples differed in education and employment status. To address this, we included these variables in the hierarchical linear regression analysis, thereby controlling for their influence.

In terms of validity, there are some weaknesses in our study: Most of the Turkish-language versions were not culturally validated. Furthermore, the issue of measurement equivalence could not be addressed due to the sample size. The multiple mediation analysis hinted at possible causal relations of the study variables. However, assumptions about causal relations cannot be confirmed using a cross-sectional, correlational design such as that implemented here, but require longitudinal or experimental studies.

The evident limitations notwithstanding, the present study contributes novel findings about treatment motivation and health beliefs of Turkish immigrant inpatients. We managed to include immigrants with a low education and poor German language proficiency in our study. This is an important yet difficult to assess population that is usually excluded from research due to language barriers.

The present study demonstrated the importance and variance of mental health beliefs, i.e., motivation for psychotherapy and illness beliefs. Turkish immigrant inpatients believed in supernatural or fatalistic causes of illness and fatalisticexternal locus of control to a greater extent than German inpatients without a migration background. These beliefs had a negative impact on motivation for psychotherapy. Consequently, they need to be addressed in psychotherapeutic treatment in order to secure adequate treatment motivation and positive treatment outcomes. More recent studies showed that mental health treatments of ethnic minority patients can be improved by cultural adaptations and by addressing culture-specific illness beliefs [78, 79]. Future research needs to develop and evaluate culturally adapted treatments for diverse populations. The challenge will be to provide feasible and realistic approaches, which can be integrated into routine mental health care. While there are endeavors towards the development of holistic treatment manuals for ethnic minority patients (e.g., [80]), the development of short modules (e.g., one-session interventions, E-Health applications, focused group sessions) could also be beneficial to complement standard treatments.

Acknowledgments We would like to thank all cooperating clinic sites, namely: Vitos Clinic for Psychiatry and Psychotherapy Marburg; Clinic for Psychiatry and Psychotherapy at the Hospital of Offenbach; Clinic for Psychiatry, Psychotherapy, and Psychosomatics at the Hospital of Frankfurt Höchst; MEDIAN "Klinik am Südpark” Bad Nauheim; and "Parkland-Klinik" Bad Wildungen.

Conflict of interest Author Hanna Reich, Author Luisa Bockel, and Author Ricarda Mewes declare that they have no conflict of interest.

Informed consent All procedures followed were in accordance with the ethical standards of the responsible committee on human experimentation (institutional and national) and with the Helsinki Declaration of 1975, as revised in 2000. Informed consent was obtained from all patients included in the study.

\section{References}

1. Bhugra D. Migration and mental health. Acta Psychiatr Scand. 2004;109:243-58. 
2. Agorastos A, Haasen C, Huber CG. Anxiety disorders through a transcultural perspective: implications for migrants. Psychopathology. 2012;45:67-77.

3. Kirmayer LJ, Narasiah L, Munoz M, Rashid M, Ryder AG, Guzder J, et al. Common mental health problems in immigrants and refugees: general approach in primary care. Can Med Assoc J. 2011;183: E959-67.

4. McDonald JT, Kennedy S. Insights into the "healthy immigrant effect": health status and health service use of immigrants to Canada. Soc Sci Med. 2004;59:1613-27.

5. Newbold KB. Self-rated health within the Canadian immigrant population: risk and the healthy immigrant effect. Soc Sci Med. 2005;60: 1359-70.

6. Aichberger MC, Schouler-Ocak M, Mundt A, Busch MA, Nickels E, Heimann HM, et al. Depression in middle-aged and older first generation migrants in Europe: results from the survey of health, ageing and retirement in Europe (SHARE). Eur Psychiatry. 2010;25: 468-75.

7. De Wit MAS, Tuinebreijer WC, Dekker J, Beekman AJTF, Gorissen WHM, Schrier AC, et al. Depressive and anxiety disorders in different ethnic groups: a population based study among native Dutch, and Turkish, Moroccan and Surinamese migrants in Amsterdam. Soc Psychiatry Psychiatr Epidemiol. 2008;43:905-12.

8. Kirmayer LJ, Young A. Culture and somatization: clinical, epidemiological, and ethnographic perspectives. Psychosom Med. 1998;60: 420-30.

9. Lindert J, Von Ehrenstein OS, Priebe S, Mielck A, Brähler E. Depression and anxiety in labor migrants and refugees - a systematic review and meta-analysis. Soc Sci Med. 2009;69:246-57.

10. Schraufnagel TJ, Wagner AW, Miranda J, Roy-Byrne PP. Treating minority patients with depression and anxiety: what does the evidence tell us? Gen Hosp Psychiatry. 2006;28:27-36.

11. Raleigh VS, Irons R, Hawe E, Scobie S, Cook A, Reeves R, et al. Ethnic variations in the experiences of mental health service users in England: results of a national patient survey programme. $\mathrm{Br} \mathrm{J}$ Psychiatry. 2007;191:304-12.

12. Arnow B, Blasey C, Manber R, Constantino MJ, Markowitz JC, Klein DN. Dropouts versus completers among chronically depressed outpatients. J Affect Disord. 2007;97:197-202.

13. Statistisches Bundesamt [Federal Statistical Office]. [Population and employment. Population with migration background - results of the microcensus 2005]. Wiesbaden 2009.

14. Eurostat. Migrants in Europe: a statistical portrait of the first and second generation. Luxembourg: European Union 2011. doi:10.2785/5318.

15. Mösko M, Schneider J, Koch U, Schulz H. [Does a Turkish migration background influence treatment outcome? Results of a prospective inpatient healthcare study]. Psychother Psych Med. 2008;58:176-82.

16. Göbber J, Pfeiffer W, Winkler M, Kobelt A, Petermann F. [Inpatient health care treatment for patients of Turkish descent-special challenges and treatment results]. Zeitschrift für Psychiatr Psychol und Psychother. 2010;58:181-7.

17. Blum L. Motivation: escape to freedom, the important ingredient in short-term psychotherapy. Psychol Rep. 1988;63:381-2.

18. Franz M, Schiessl N, Manz R, Fellhauer R, Schepank H, Tress W. [Psychotherapy motivation and psychotherapy acceptance. Initial results of the Mannheim intervention study]. Psychother Psychosom Med Psychol. 1990;40:369-74.

19. Keithly LJ, Samples SJ, Strupp HH. Patient motivation as a predictor of process and outcome in psychotherapy. Psychother Psychosom. 1980;33:87-97.

20. Sifneos PE. Motivation for change a prognostic guide for successful psychotherapy. Psychother Psychosom. 1978;29:293-89.

21. Schneider W, Klauer T. Symptom level, treatment motivation, and the effects of inpatient psychotherapy. Psychother Res. 2001;11:153-67.

22. Nickel C, Muehlbacher M, Kettler C, Tritt K, Egger C, Lahmann C. [Treatment motivation and results of inpatient psychotherapy for women with depressive disorders: a prospective study]. Gesundheitswesen. 2006;68:11-7.

23. Klauer T, Maibaum F, Schneider W. [Patient and therapist reports of treatment motivation as predictors of dropout from inpatient psychotherapy]. Zeitschrift für Psychiatr Psychol und Psychother. 2007;55:59-66.

24. Calliess IT, Schmid-Ott G, Akguel G, Jaeger B, Ziegenbein M. [Attitudes towards psychotherapy of young second-generation Turkish immigrants living in Germany]. Psychiatr Prax. 2007;34:343-8.

25. Drieschner KH, Lammers SM, van der Staak CP. Treatment motivation: an attempt for clarification of an ambiguous concept. Clin Psycholology Rev. 2004;23:1115-37.

26. Petrie KJ, Weinman J. Patients' perceptions of their illness: the dynamo of volition in health care. Curr Dir Psychol Sci. 2012;21:60-5.

27. Bhugra D. Cultural identities and cultural congruency: a new model for evaluating mental distress in immigrants. Acta Psychiatr Scand. 2005; 111:84-93.

28. Kirmayer LJ, Sartorius N. Cultural models and somatic syndromes. Psychosom Med. 2007;69:832-40.

29. Kleinmann A. Patients and healers in the context of culture: an exploration of the borderland between anthropology, medicine, and psychiatry. Berkeley: University of California Press; 1980.

30. Bowling A. Beliefs about illness causation among Turkish and white British people living in a deprived inner London district. Health Educ Res. 1994;9:355-64.

31. Bäärnhielm S, Ekblad S. Turkish migrant women encountering health care in Stockholm: a qualitative study of somatization and illness meaning. Cult Med Psychiatry. 2000;24:431-52.

32. Franz M, Lujic C, Koch E, Wüsten B, Yürük N, Gallhofer B. [Subjective illness beliefs of Turkish migrants with mental disorders-specific characteristics compared to German patients]. Psychiatr Prax. 2007;34:332-8.

33. Kizilhan J. [Interaction of illness perception and coping with Turkish patients - a comparative study]. Verhal Verhal. 2008;29:352-66.

34. Karanci NA. Causal attributions for psychological illness among Turkish psychiatric in-patients and their relationships with hope. Int J Soc Psychiatry. 1986;32:3-12.

35. Rosenbaum R, Horowitz M. Motivation for psychotherapy: a factorial and conceptual analysis. Psychotherapy. 1983;20:346-54.

36. Autorengruppe Bildungsberichterstattung [author group educational reports]. Education in Germany 2012: An indicator-based report including analysis of cultural education in the life course. Bertelsmann: Bielefeld; 2012.

37. Woellert F, Kröhnert S, Sippel L, Klingholz R. Unused potentials: Current State of Integration in Germany. Berlin-Institut für Bevölkerung und Entwicklung [Berlin Institute for Population and Development] 2009. doi:10.1007/978-3-531-19010-5 8

38. Statistisches Bundesamt [Federal Statistical Office]. [Population and employment. Population with migration background - results of the microcensus 2010]. Wiesbaden: Statistisches Bundesamt 2011.

39. Erim Y, Morawa E, Özdemir DF, Senf W. [Prevalence, comorbidity and severity of psychosomatic disorders in outpatients with Turkish migration background]. Psychother Psychosom Medizinische Psychol. 2011;61:472-80.

40. Brislin RW, Lonner WJ, Throndike RM. Cross cultural research methods. New York: Wiley; 1973.

41. Schneider W, Basler HD, Beisenherz B. Questionnaire for the measurement of psychotherapy motivation: FMP. Beltz-Test: Weinheim; 1989.

42. Kroenke K, Spitzer RL, Williams JBW. The PHQ-9: validity of a brief depression severity measure. Prim Care. 2001;16:606-13.

43. Kroenke K, Spitzer RL, Williams JBW. The PHQ-15: validity of a new measure for evaluating the severity of somatic symptoms. Psychosom Med. 2002;64:258-66.

44. Kroenke K, Spitzer R, Williams J, Löwe B. The patient health questionnaire somatic, anxiety, and depressive symptom scales: a systematic review. Psychiatry Prim Care. 2010;32:345-59. 
45. Henkel V, Mergl R, Kohnen R, Maier W, Möller HJ, Hegerl U. Identifying depression in primary care: a comparison of different methods in a prospective cohort study. BMJ. 2003;326:200-1.

46. Wittkampf K, Naeije L, Schene A, Huyser J, van Weert H. Diagnostic accuracy of the mood module of the patient health questionnaire: a systematic review. Psychiatry Prim Care. 2007;29:388-95.

47. Löwe B, Spitzer RL, Zipfel S, Herzog W. Gesundheitsfragebogen für Patienten [Health questionnaire for patients]. Karlsruhe: Pfizer; 2002.

48. Çorapçığlu A, Özer GU. Hasta sağılık anketi-9. Patient Heal. Quest. Screeners. http://www.phqscreeners.com/pdfs/02_PHQ-9/PHQ9_ Turkish for Turkey.pdf. Accessed 9 May 2012.

49. Pfizer Inc. Patient Health Questionnaires (PHQ) Screeners, official website. 2013.http://www.phqscreeners.com/overview.aspx? Screener=02_PHQ-9. Accessed 30 Aug 2013.

50. Yazici Güleç M, Güleç H, Simșek G, Turhan M, Aydin SE. Psychometric properties of the Turkish version of the patient health questionnaire - somatic, anxiety, and depressive symptoms. Compr Psychiatry. 2012;53:623-9.

51. Kroenke K, Spitzer RL, DeGruy FV, Swindle R. A symptom checklist to screen for somatoform disorders in primary care. Psychosomatics. 1998;39:263-72.

52. Broadbent E, Petrie KJ, Main J, Weinman J. The brief illness perception questionnaire. J Psychosom Res. 2006;60:631-7.

53. Moss-Morris R, Weinman J, Petrie K, Horne R, Cameron L, Buick D. The revised illness perception questionnaire (IPQ-R). Psychol Heal. 2002;17:1-16.

54. Oflaz SB. Kısa hastalık alğı ölçeği. http://www.uib.no/ipq/. Accessed 9 May 2012

55. Rief W. Fragebogen zur Krankheitswahrnehmung (Kurzform). http:// www.uib.no/ipq/. Accessed 9 May 2012.

56. Gaab J. Krankheitsannahmen (Illness Perception Questionnaire - R). http://www.uib.no/ipq/index.html. Accessed 9 May 2012.

57. Lohaus A, Schmitt GM. Locus of control inventory for illness and health. Göttingen: Hogrefe; 1989.

58. Cohen J. Statistical power analysis for the behavioral sciences. 2nd ed. Hillsdale: Erlbaum; 1988

59. Grissom RJ, Kim JJ. Effect sizes for research: univariate and multivariate applications. 2nd ed. New York,: Taylor \& Francis; 2012.

60. Preacher KJ, Hayes AF. Asymptotic and resampling strategies for assessing and comparing indirect effects in multiple mediator models. Behav Res Methods. 2008;40:879-91.

61. Mallinckrodt B, Abraham WT, Wei M, Russell DW. Advances in testing the statistical significance of mediation effects. J Couns Psychol. 2006;53:372-8.

62. Ferber L V, Köster I, Celayir-Erdogan N. [Turkish and German patients of general practitioners - diseases, drug expectations and drug prescriptions]. Gesundheitswesen. 2003;65:304-11.

63. Mocan-Aydin G. Western models of counseling and psychotherapy within Turkey: crossing cultural boundaries. Couns Psychol. 2000;28:281-98.

64. Machleidt W, Behrens K, Ziegenbein M. [Integration of immigrants into the mental health care system in Germany]. Psychiatr Prax. 2007;34:325-31.
65. Glaesmer H, Wittig U, Braehler E, Martin A, Mewes R, Rief W. Health care utilization among first and second generation immigrants and native-born Germans: a population-based study in Germany. Int $\mathrm{J}$ Public Health. 2011;56:541-8.

66. Raney S, Çınarbas D. Counseling in developing countries: Turkey and India as examples. J Ment Heal Couns. 2005;27: 149-60.

67. Eskin M. Rural population's opinions about the causes of mental illness, modern psychiatric help-sources and traditional healers in Turkey. Int J Soc Psychiatry. 1989;35:324-8.

68. Minas H, Klimidis S, Tuncer C. Illness causal beliefs in Turkish immigrants. BMC Psychiatry. Published Online First: 2007. doi:10. 1186/1471-244X-7-34.

69. Tuncer C. Mental illness in an Islamic-Mediterranean culture: Turkey. In: Al-Issa I, ed. Handbook of culture and mental illness: an international perspective. Madison, CT: International Universities Press 1995. 169-82.

70. Lester D, Castromayor IJ, Içli T. Locus of control, depression, and suicidal ideation among American, Philippine, and Turkish students. J Soc Psychol. 1991;131:447-9.

71. Ergüder Ü, Esmer Y, Kalaycioğlu E. Türk toplumunun degáerleri [Values inTurkish culture]. Istanbul, Turkey: Tüsiad Yayinlari, Tüsiad, publication number T/91, 6.1451991.

72. Krause MS. A cognitive theory of motivation for treatment. J Gen Psychol. 1966;75:9-19.

73. Miller WR. Motivation for treatment: a review with special emphasis on alcoholism. Psychol Bull. 1985;98:84-107.

74. Raskin A. Factors therapists associate with motivation to enter psychotherapy. J Clin Psychol. 1961;17:62-5.

75. Fassaert T, de Wit MAS, Tuinebreijer WC, Verhoeff AP, Beekman ATF, Dekker J. Perceived need for mental health care among nonwestern labour migrants. Soc Psychiatry Psychiatr Epidemiol. 2009;44:208-16.

76. Behrens K, Machleidt W, Haltenhof H, Ziegenbein M, Calliess IT. [Somatization and vulnerability to offence in immigrants with mental disorders — evidence or eminence?]. Nervenheilkunde. 2008;27:63943.

77. Mewes R, Rief W. [Are somatoform complaints and causal attributions in Turkish migrants associated with their cultural background or the migration itself?]. Zeitschrift für Medizinische Psychol. 2009;18: $135-9$.

78. Benish SG, Quintana S, Wampold BE. Culturally adapted psychotherapy and the legitimacy of myth: a direct-comparison meta-analysis. J Couns Psychol. 2011;58:279-89.

79. Van Loon A, van Schaik A, Dekker J, Beekman A. Bridging the gap for ethnic minority adult outpatients with depression and anxiety disorders by culturally adapted treatments. J Affect Disord. 2013;147:9-16.

80. Hinton DE, Rivera EI, Hofmann SG, Barlow DH, Otto MW. Adapting CBT for traumatized refugees and ethnic minority patients: examples from culturally adapted CBT (CA-CBT). Transcult Psychiatry. 2012;49:340-65. 